Received: 2014.11.25 Accepted: 2015.02.20 Published: 2015.05.25

Authors' Contribution: Study Design A Data Collection B Statistical Analysis C Data Interpretation D Manuscript Preparation E Literature Search F Funds Collection G
Corresponding Author: Source of support:

\section{Technical Refinement of Hepatic Vein Reconstruction in Living Donor Liver Transplantation Using Left Liver Graft}

Department of Surgery, Nagasaki University Graduate School of Biomedical Sciences, Nagasaki, Japan

Background: In adult living donor liver transplantation (LDLT), left liver graft is generally safer for the donor. The aim of this study was to demonstrate a technical refinement for achieving sufficient outflow using left liver graft.

Material/Methods: Forty-seven cases using left liver were divided into 2 groups according to the procedures of hepatic vein reconstruction: the side-clamp group (21 cases), and the cross-clamp group (26 cases), to sufficiently enlarge the diameter of the hepatic vein with excising the inferior vena cava (IVC).

Results: The liver function tests at 7 days after LDLT were not significantly different between the 2 groups, but the median amount of ascites was significantly greater in the side-clamp group (1250 ml; range, 484-3690) than in the cross-clamp group (582 $\mathrm{ml}$; 190-2785). When we selected the patients with the ratio of graft weight to recipient standard liver volume less than 30\%, the 1-year patient survival after transplantation was significantly better in the cross-clamp group than in the side-clamp group ( $90 \%$ in cross-clamp group vs. $71 \%$ in side-clamp group, $\mathrm{P}<0.05)$.

Conclusions: In conclusion, hepatic vein reconstruction with cross-clamping of the IVC can secure a sufficient outflow in LDLT using left liver graft.

MeSH Keywords: Hepatic Veins • Liver Transplantation • Living Donors

Abbreviations: $\quad$ LDLT - living donor liver transplantation; SFSS - small-for-size syndrome; MHV - middle hepatic vein; LHV - left hepatic vein; IVC - inferior vena cava; RHV - right hepatic vein; CECT - contrast-enhanced computed tomography; MELD - model of end-stage liver disease; GW/RSLV - ratio of graft weight to recipient standard liver volume

Full-text PDF: http://www.annalsoftransplantation.com/abstract/index/idArt/893136 


\section{Background}

In living donor liver transplantation (LDLT), the recipients sometimes encounter small-for-size syndrome (SFSS), with clinical manifestations of massive ascites and prolonged jaundice because of relatively excessive portal flow into insufficient graft liver volume [1]. SFSS can ultimately lead to graft failure, so several technical inventions have been introduced, mainly to decrease portal flow, such as porto-caval shunting [2], splenic artery ligation [3], or splenectomy [4]. However, as a matter of course, securing optimal outflow should be equally important to overcoming SFSS.

In regard to the donor selection in LDLT, several reports have introduced the possible advantages of left liver graft with the middle hepatic vein (MHV) (extended left lobe graft) over right liver graft, because although the actual volume is smaller in the left liver graft, the incidence of severe postoperative complications and mortality are greater in donors providing right liver graft than left liver graft [5] but the recipient outcome is not significantly different [6]. Accordingly, our current policy is to use the left liver graft as a first line because donor safety is the top priority in LDLT. Especially in LDLT using left liver graft, it is important to develop technical refinements to overcome SFSS.

The aim of this study was to show our new technique to achieve adequate outflow in LDLT using left liver graft.

\section{Material and Methods}

\section{Patients}

Of the 102 LDLT patients in Nagasaki University from April 2005 to May 2011, 47 adult LDLT cases using left liver graft with MHV were enrolled in this study. Initially, we preferred to use right liver graft in adult LDLT, but from April 2005 we changed our policy to use the left liver graft as the first-line. They were divided into 2 groups: a side-clamp group in which the hepatic vein was reconstructed with the conventional procedure of side-clamping the recipient MHV/left hepatic vein (LHV) ( $n=21$, from April 2005 to May 2009), and a cross-clamp group in which our new and current procedure of cross-clamping the suprahepatic inferior vena cava (IVC) was performed $(n=26$, from June 2009 to May 2011).

\section{Surgical techniques (Figure 1)}

During the recipient operation, the right hepatic vein (RHV) was divided using a vascular stapling device [7]. After the portal triad was divided at the hepatic hilum, the whole liver was removed with dividing the MHV and LHV, which were clamped with vascular clamps. During the anhepatic phase, the tissue surrounding the MHV/LHV was carefully dissected, and the suprahepatic IVC just beyond the MHV/LHV was clamped last. After the infrahepatic IVC was also clamped, cavotomy was performed to make a large orifice for anastomosis, excising the right corner of the MHV longitudinally.

In the side-clamp group, the common trunk of the MHV/LHV was clamped, and hepatic vein reconstruction was performed after dividing the septum between the MHV/LHV.
A
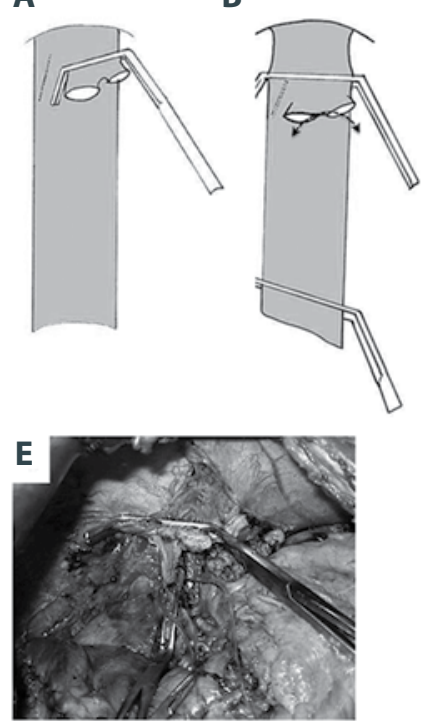

C

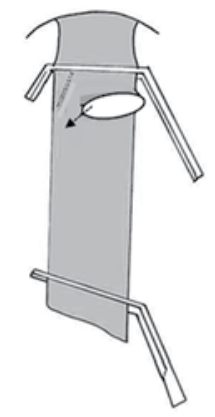

$\mathbf{F}$

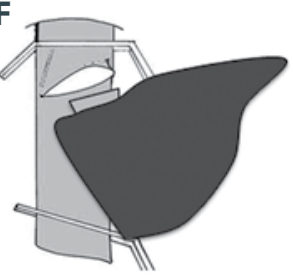

D

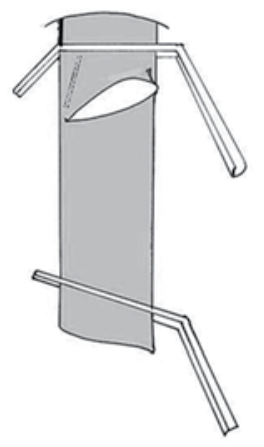

Figure 1. Procedure of hepatic vein reconstruction with IVC crossclamping. At the final step of total hepatectomy of the recipient, the left hepatic vein (LHV) and middle hepatic vein (MHV) were divided with clamping of their common trunk (A). After the IVC was cross-clamped, the septum between the LHV and MHV was divided (B), and the right corner of MHV was excised horizontally (C) to create a larger orifice for anastomosis (D). Actual macroscopic finding of the procedure (E). The left liver graft was put in place, and $\mathrm{HV}$ reconstruction was performed with sufficient space for anastomosis (F). 
In regard to the MHV/LHV of the graft, venoplasty was generally performed to make 1 orifice when the common trunk between MHV and LHV was short, or their orifices were independently divided [8]. Briefly, the septum between the MHV and LHV was divided longitudinally and sutured horizontally using 5-0 monofilament non-absorbable suture.

In both groups, hepatic vein anastomosis was performed using 4-0 non-absorbable monofilament suture.

Splenectomy was performed in the selected cases with platelet count less than or equal to $5000 / \mu \mathrm{l}$ or HCV-cirrhosis cases to collect platelets for subsequent interferon therapy after LDLT.

\section{Postoperative care}

Basic immunosuppression after transplantation consisted of tacrolimus and steroid.

Doppler ultrasound was performed daily to check the patency of vascular anastomosis in portal vein, hepatic vein, and hepatic artery until 7 days after LDLT and when indicated thereafter. Hepatic venous flow was considered as good when the wave form was biphasic or triphasic, and actual measurement of flow volume or velocity was not routinely performed. Contrast-enhanced computed tomography (CECT) of the liver was performed routinely at 7 days after LDLT, as long as the renal function was normal. We have digital data of imaging studies on computed medical charts from 2005, which made it possible for us to measure the diameter of the HV anastomosis accurately.

An abdominal drain was generally placed at the right subcostal space during surgery, and removed when the amount of ascites became less than $500 \mathrm{ml} /$ day.

\section{Analyses}

The liver function tests, the amount of ascites before and after LDLT, the diameter of HV anastomosis on CECT, and patient/graft function after LDLT were compared between the 2 groups. The amount of ascites before LDLT was classified into 3 grades based on CT finding: none, minimal (visualized only at liver surface or pelvic space), and severe (visualized whole abdomen).

Statistical analyses were performed using the Mann-Whitney $\mathrm{U}$ test for continuous data, and the chi-square test for categorical data. Overall survival was calculated with the KaplanMeier method, and data were compared with the log-rank test. We considered $P$ values $<0.05$ to be statistically significant.

\section{Results}

\section{Patient characteristics and surgical outcome}

The patient characteristics and surgical outcomes are summarized in Table 1. Recipient/donor ages, sex ratios, and preoperative model of end-stage liver disease (MELD) scores, ChildPugh classification, total ischemia time (from vascular division in the donor to reperfusion in the recipient), the amount of ascites on CT before LDLT, and the degree of graft steatosis at time-zero biopsy were not significantly different between the groups. The surgery duration was shorter and intraoperative blood loss was less in the cross-clamp group compared to the side-clamp group, possibly because of the learning curve. Both groups each included 1 Child-Pugh grade A case of Caroli's disease, both of which underwent LDLT, not for decompensated cirrhosis, but for refractory cholangitis. In regard to the graft size, there was no significant difference between the groups for the median ratio of graft weight to recipient standard liver volume (GW/RSLV) - 35.3\% (range, 24.8-48.0) in the sideclamp group vs. $33.7 \%$ (range, 22.5-46.6) in the cross-clamp group. The incidence of splenectomy was not significantly different between the groups - 12 cases $(61.9 \%)$ in the sideclamp group vs. 20 cases $(76.9 \%)$ in the cross-clamp group.

\section{Postoperative liver functions, the amount of ascites, diameter of HV anastomosis, and graft survival}

All patients in the cross-clamp group tolerated the procedure, without any significant hypotension during surgery or postoperative renal dysfunction. In most cases in the cross-clamp group, hepatic venous flow was excellent with triphasic wave form on Doppler ultrasound just after reperfusion (Figure 2).

Overall, the median prothrombin time and total bilirubin at 7 days after transplantation were not significantly different between the 2 groups, but the median amount of ascites was significantly less in the cross-clamp group $(582 \mathrm{ml}$; range, 190-2785) than in the side-clamp group (1250 $\mathrm{ml}$; range, 484-3690) $(\mathrm{P}<0.01$, Mann-Whitney test) (Figure 3).

In an analysis of patients with extremely small graft size (GW/RSLV $<30 \% ; 7$ in the side-clamp group, 10 in the crossclamp group), the median prothrombin time was higher $(P=0.106)$ in the cross-clamp group (49.0\%; range, $28.0-66)$ than in the side-clamp group (39.0\%; range, 25.0-53.0); the median total bilirubin was lower $(P=0.079)$ in the cross-clamp group (3.8 $\mathrm{mg} / \mathrm{dl}$; range, 1.7-17.0) than in the side-clamp group (7.5 $\mathrm{mg} / \mathrm{dl}$; range, 1.7-14.5); and the median amount of ascites was less $(P=0.184)$ in the cross-clamp group $(628 \mathrm{ml} /$ day; range, 209-2560) than in the side-clamp group (1060 ml/day; range, 484-3040), but these differences did not reach statistical significance (Figure 4). 
Table 1. Patient characteristics and surgical outcomes.

\begin{tabular}{|c|c|c|c|}
\hline & $\begin{array}{l}\text { Side clamp } \\
\qquad(n=21)\end{array}$ & $\begin{array}{l}\text { Cross clamp } \\
\qquad(n=26)\end{array}$ & \\
\hline Recipient age & $55(25-68)$ & $57(27-72)$ & NS \\
\hline Donor age & $33(19-64)$ & $33(20-65)$ & NS \\
\hline Gender & Male 11 Female 10 & Male 12 Female 14 & NS \\
\hline MELD & $16(7-30)$ & $15(7-42)$ & NS \\
\hline \multicolumn{4}{|l|}{ Child-Pugh } \\
\hline A & 1 & 1 & \multirow{3}{*}{ NS } \\
\hline B & 10 & 11 & \\
\hline C & 10 & 14 & \\
\hline \multicolumn{4}{|l|}{ Preoperative ascites } \\
\hline None & 8 & 10 & \multirow{3}{*}{ NS } \\
\hline Minimal & 9 & 11 & \\
\hline Severe & 4 & 5 & \\
\hline \multirow{2}{*}{ Duration of surgery (min) } & 915 & 746 & \multirow{2}{*}{$P<0.01$} \\
\hline & $(690-1,159)$ & $(578-999)$ & \\
\hline \multirow{2}{*}{ Total ischemic time (min) } & 168 & 154 & \multirow{2}{*}{ NS } \\
\hline & $(84-219)$ & $(107-282)$ & \\
\hline \multirow{2}{*}{ Blood loss (g) } & 5450 & 3800 & \multirow{2}{*}{$P<0.05$} \\
\hline & $(1,350-26,876)$ & $(980-17,600)$ & \\
\hline \multirow{2}{*}{ GW/RSLV (\%) } & 35,3 & 33,7 & \multirow{2}{*}{ NS } \\
\hline & $(24.8-48.0)$ & $(22.5-46.6)$ & \\
\hline Graft stetatosis (\%) & $5(0-50)$ & $5(0-50)$ & NS \\
\hline Splenectomy & 12 & 20 & NS \\
\hline
\end{tabular}

Data are expressed as median (range); MELD - model of end-stage liver disease; GW - graft weight; RSLV - recipient standard liver volume; NS - not significant.
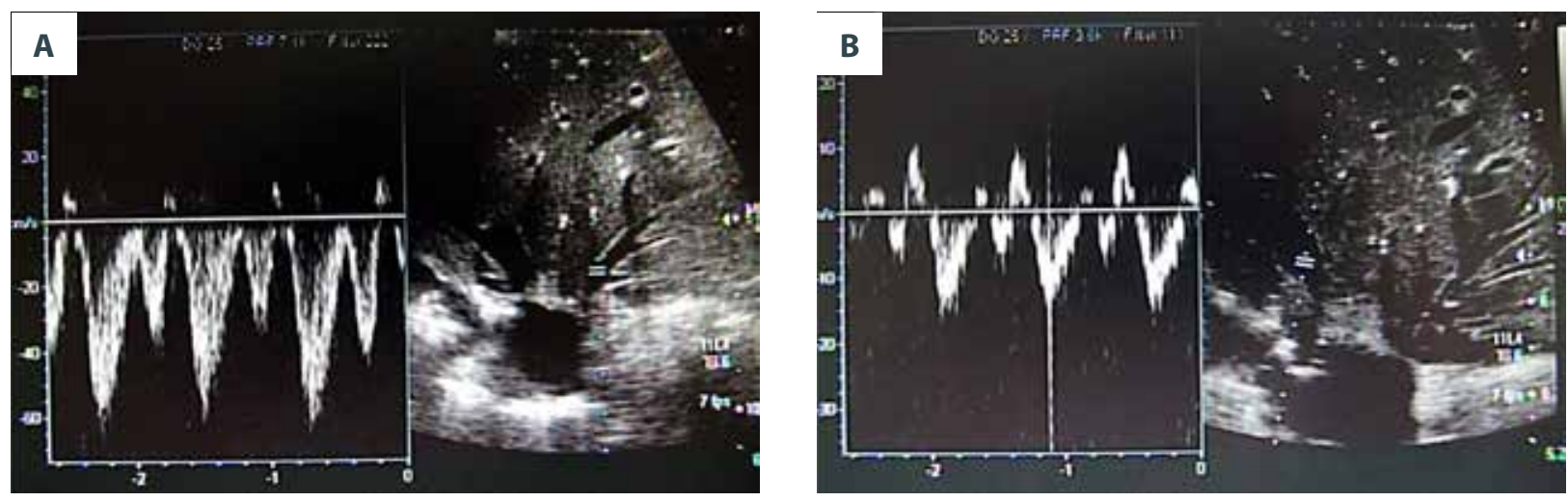

Figure 2. Doppler ultrasound just after reperfusion of the left liver graft. Hepatic venous flow was excellent with triphasic wave form both in LHV (A) and MHV (B). 

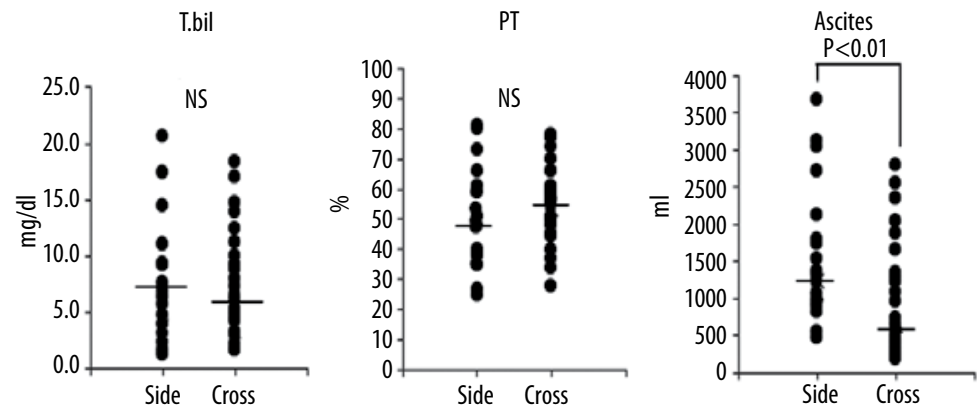

Figure 3. The level of total bilirubin, prothrombin time, and the amount of ascites 7 days after LDLT in the sideclamp group $(\mathrm{n}=21)$ and cross-clamp group $(n=26)$. The amount of ascites was significantly less in the crossclamp group.
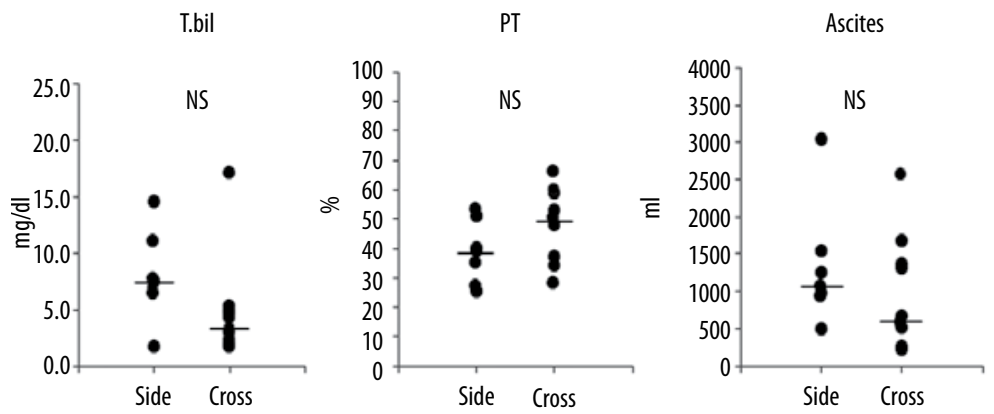

Figure 4. The level of total bilirubin, prothrombin time, and the amount of ascites 7 days after LDLT in the cases with extremely small graft size (GW/RSLV $<30 \%$; 7 in the side-clamp group and 10 in the cross-clamp group).
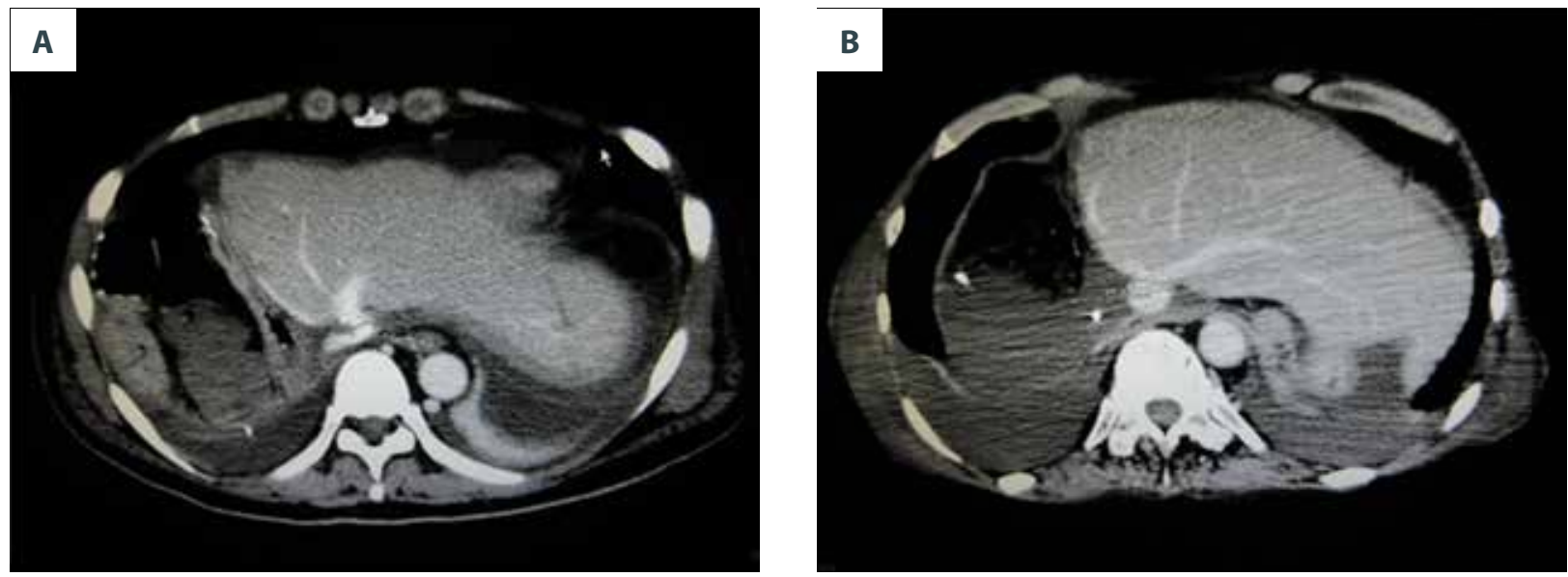

Figure 5. The diameter of HV anastomosis on CECT at 7 days after LDLT, in a case with side-clamp (A, $10.56 \mathrm{~mm}$ ) and cross-clamp (B, $15.47 \mathrm{~mm}$ ).

In the patients with extremely small graft size, the 1-year patient/graft survival was significantly better in the cross-clamp group than in the side-clamp group ( $90 \%$ vs. $71 \%$; $P<0.05$, log rank test). Two patients died of graft failure 50 days and 38 days after LDLT in the side-clamp group, while 1 patient died of systemic candida infection 19 days after LDLT in the crossclamp group. Of 2 patients who died of graft failure in the side-clamp group, 1 had bleeding of the esophageal varices without portal vein obstruction, and 1 had complicated sepsis; neither of these appeared to be directly related to SFSS.
CECT could be performed at 7 days after LDLT in 15 of 21 patients $(71 \%)$ with side-clamp, and 19 of 26 (73\%) with crossclamp. The median diameter of HV anastomosis at 7 days after LDLT on CECT was significantly larger $(P<0.05)$ in the crossclamp group (14.8 $\mathrm{mm}$; range, 6.9-19.5) than in the side-clamp group (10.9 mm; range, 5.5-14.6) (Figure 5).

\section{Discussion}

It is important to control the balance between the portal inflow and hepatic vein outflow of the liver to maintain hepatocyte 
function. In general, portal circulation is hyper-dynamic in cirrhotic patients [9]; therefore, modulation of portal inflow is recognized as an effective procedure to overcome SFSS in LDLT. The balance of blood flow, as well as steatotic liver and older donor age, can affect SFSS, but blood flow is the only factor that can be controlled surgically. However, a few studies have demonstrated the importance of the outflow reconstruction from the point of view of avoiding SFSS. In LDLT cases using right liver graft, the reconstruction of MHV tributaries or right inferior hepatic vein have been well documented to avoid graft congestion [10,11]. As mentioned before, the outcome of LDLT recipients using a left liver graft is reported to be not different from that using a right liver graft in spite of the fact that left liver volume is generally smaller than right liver volume, possibly because the whole left liver is well drained via the middle and left hepatic veins. Accordingly, hepatic vein reconstruction is important to secure the sufficient outflow in LDLT using left liver graft. In this study, creating a larger orifice by crossclamping the IVC proved to be an effective method to overcome SFSS with decreasing ascites, especially in cases of extremely small graft size (GW/RLSV $<30 \%)$. Although differences in several factors, including total bilirubin, prothrombin time, and amount of ascites after LDLT, did not reach statistical significance (possibly because of small sample size), postoperative liver functions seemed better, and 1-year patient survival was significantly better in the cross-clamp group.

Actually, this procedure of HV reconstruction with crossclamping is not novel. Tanaka et al. reported this procedure in selected pediatric LDLT cases more than 20 years ago [12], emphasizing that it is important to make a large orifice in IVC to avoid twisting at the HV anastomosis. In our study, the large diameter of the HV anastomosis was maintained better compared to the side-clamp group 7 days after LDLT. We believe that a large orifice with excising the IVC can produce long and short anastomosis of HV, and it contributes to sufficient venous outflow without twisting the anastomosis. Actually, measurement of portal and/or hepatic vein flow might be better to show the efficacy of the HV reconstruction with IVC cross-clamping, but unfortunately we did not routinely check the quantity of the portal/HV flows. As the objective analysis, we checked the diameter of HV anastomosis on CECT after LDLT.

\section{References:}

1. Hori T, Ogura Y, Ogawa K et al: How transplant surgeons can overcome the inevitable insufficiency of allograft size during adult living-donor liver transplantation: strategy for donor safety with a smaller-size graft and excellent recipient results. Clin Transplant, 2012; 26: E324-34

2. Boillot O, Delafosse B, Méchet I et al: Small-for-size partial liver graft in an adult recipient; a new transplant technique. Lancet, 2002; 359: 406-7

3. Graham JA, Samstein B, Emond JC, Kato T: Functional distal splenorenal shunt and splenic artery ligation as portal flow modulation in left lobe living donor transplantation. Liver Transpl, 2014; 20: 245-46
Hypotension and/or postoperative renal dysfunction are potential concerns associated with cross-clamping IVC, but there were no cases that developed significant adverse effects, and all patients tolerated the procedure well, with sufficient fluid infusion.

Technically, because we used a vascular stapler to divide the right hepatic vein, the excision of the IVC was safely performed without any risk of cutting the continuous suture at the right hepatic vein, and sufficient space for anastomosis could be obtained by clamping the IVC, even in deep and small surgical spaces in adult cases.

This technique of cross-clamping the IVC can be adopted also in LDLT using a right lobe graft, especially in complex cases such as those requiring the reconstruction of multiple middle hepatic vein tributaries or right inferior hepatic veins. In our series, 2 cases of right lobe LDLT underwent multiple hepatic vein reconstruction successfully with cross-clamping of the IVC during the study period (data not shown).

Limitations of this study include its small sample size and the fact that it was a comparison study using historical control. Because the MELD score and Child-Pugh grade before LDLT were not different between the groups, the better outcome in the cross-clamp group might be related to just the learning curve, because the duration of surgery and blood loss were significantly less in the cross-clamp group. Also, in the cases of extremely small graft size, the liver functions and the amount of ascites did not reach statistically significant differences between the cross-clamp group and the side-clamp group. Although we believe a large orifice of HV anastomosis with IVC cross-clamping is theoretically feasible, the actual efficacy of cross-clamping needs to be demonstrated in a prospective randomized control study.

\section{Conclusions}

In conclusion, although a randomized study is needed to determine the actual advantage of this procedure, we have shown that creating a larger orifice with cross-clamping the IVC is a safe and effective procedure to overcome SFSS in LDLT using left liver graft.

4. Yoshizumi T, Taketomi A, Soejima Y et al: The beneficial role of simultaneous splenectomy in living donor liver transplantation in patients with small-for-size graft. Transpl Int, 2008; 21: 833-42

5. Umeshita K, Fujiwara K, Kiyosawa K et al: Japanese Liver Transplantation Society. Operative morbidity of living liver donors in Japan. Lancet, 2003; 362: 687-90

6. Soejima Y, Shirabe K, Taketomi A et al: Left lobe living donor liver transplantation in adults. Am J Transplant, 2012; 12: 1877-85 
7. Eguchi S, Kawashita Y, Takatsuki M, Kanematsu T: Application of endovascular stapler in living-donor liver transplantation. Am J Surg, 2007; 193: 258-59

8. Concejero A, Chen CL, Wang CC et al: Donor graft outflow venoplasty in living donor liver transplantation. Liver Transpl, 2006; 12: 264-68

9. Hori T, Yagi S, lida T et al: Stability of cirrhotic systemic hemodynamics ensures sufficient splanchnic blood flow after living-donor liver transplantation in adult recipients with liver cirrhosis. World J Gastroenterol, 2007; 13: 5918-25
10. Yi NJ, Suh KS, Lee HW et al: An artificial vascular graft is a useful interpositional material for drainage of the right anterior section in living donor liver transplantation. Liver Transpl, 2007; 13: 1159-67

11. Hwang S, Ha TY, Ahn CS et al: Reconstruction of inferior right hepatic veins in living donor liver transplantation using right liver grafts. Liver Transpl, 2012; 18: 238-47

12. Tanaka K, Uemoto S, Tokunaga $Y$ et al: Surgical techniques and innovations in living related liver transplantation. Ann Surg, 1993; 217: 82-91 